ARTICLE

Received 18 Jul 2014 | Accepted 28 Nov 2014 | Published 19 Jan 2015

DOl: $10.1038 /$ ncomms 6979

\title{
Stable metal-organic frameworks containing single-molecule traps for enzyme encapsulation
}

\author{
Dawei Feng ${ }^{1, \star}$, Tian-Fu Liu ${ }^{1, \star}$, Jie Su ${ }^{2}$, Mathieu Bosch ${ }^{1}$, Zhangwen Wei ${ }^{1}$, Wei Wan ${ }^{2}$, Daqiang Yuan ${ }^{3}$, \\ Ying-Pin Chen ${ }^{1,4}$, Xuan Wang ${ }^{1}$, Kecheng Wang${ }^{1}$, Xizhen Lian', Zhi-Yuan Gu1', Jihye Park1, \\ Xiaodong Zou ${ }^{2} \&$ Hong-Cai Zhou ${ }^{1,4}$
}

Enzymatic catalytic processes possess great potential in chemical manufacturing, including pharmaceuticals, fuel production and food processing. However, the engineering of enzymes is severely hampered due to their low operational stability and difficulty of reuse. Here, we develop a series of stable metal-organic frameworks with rationally designed ultra-large mesoporous cages as single-molecule traps (SMTs) for enzyme encapsulation. With a high concentration of mesoporous cages as SMTs, PCN-333(Al) encapsulates three enzymes with record-high loadings and recyclability. Immobilized enzymes that most likely undergo single-enzyme encapsulation (SEE) show smaller $K_{m}$ than free enzymes while maintaining comparable catalytic efficiency. Under harsh conditions, the enzyme in SEE exhibits better performance than free enzyme, showing the effectiveness of SEE in preventing enzyme aggregation or denaturation. With extraordinarily large pore size and excellent chemical stability, PCN-333 may be of interest not only for enzyme encapsulation, but also for entrapment of other nanoscaled functional moieties.

\footnotetext{
${ }^{1}$ Department of Chemistry, Texas A\&M University, College Station, Texas 77843-3255, USA. ${ }^{2}$ Berzelii Centre EXSELENT on Porous Materials and Inorganic and Structural Chemistry, Department of Materials and Environmental Chemistry, Stockholm University, 10691 Stockholm, Sweden. ${ }^{3}$ State Key Laboratory of Structural Chemistry, Fujian Institute of Research on the Structure of Matter, Chinese Academy of Sciences, Fujian, Fuzhou 350002, P. R. China. ${ }^{4}$ Department of Materials Science and Engineering, Texas A\&M University, College Station, Texas 77842, USA. * These authors contributed equally to this work. Correspondence and requests for materials should be addressed to H.-C.Z. (email: zhou@chem.tamu.edu).
} 
M etal-organic frameworks (MOFs) with large pores or channels are considered to be promising candidates for the immobilization of many catalysts, such as metal complexes, nanoparticles and enzymes ${ }^{1-4}$. Immobilization is particularly valuable for enzymes, since they are costly and can be difficult to reuse and recover ${ }^{5}$. A range of solid supports have been explored for enzymes, such as sol gels, hydrogels, organic microparticles, and porous or non-porous inorganic supports ${ }^{5}$. However, there have been disadvantages associated with these supports, including very low protein loading in the non-porous systems and denaturation of enzymes in sol gels due to their brittle and disordered structures ${ }^{5-9}$. Mesoporous silica nanoparticles, which have a large pore size, high porosity and a very ordered structure, have been demonstrated as promising enzyme supports ${ }^{5}$. However, due to their one-dimensional (1D) channel structure, insufficient interactions between the inner channel surface of mesoporous silica and enzymes result in significant leaching during multiple cycles of use. Moreover, aggregation still occurs inside the channel ${ }^{5-9}$.

Recently, we studied MOFs containing predesigned singlemolecule traps (SMTs) for carbon capture, wherein each SMT can trap one $\mathrm{CO}_{2}$ molecule ${ }^{10}$. If the same concept is applied in enzyme immobilization, we can potentially diminish leaching and encapsulate each enzyme molecule in a SMT to eliminate aggregation, optimizing the catalytic environment. MOFs, which have ultra-high porosity, tunable pore shape and size and adjustable surface functionality, are an ideal platform for this novel single-enzyme encapsulation (SEE) or multiple-enzyme encapsulation (MEE) approach.

On the basis of structural rationalization, we herein report facile syntheses of a series of highly stable SMT-containing MOFs based on trivalent metal species, namely PCN-332(M) $(\mathrm{M}=$ $\mathrm{Al}(\mathrm{III}), \mathrm{Fe}(\mathrm{III}), \mathrm{Sc}(\mathrm{III}), \mathrm{V}(\mathrm{III}), \mathrm{In}(\mathrm{III}))$ and $\mathrm{PCN}-333(\mathrm{M})(\mathrm{M}=$ $\mathrm{Al}(\mathrm{III}), \mathrm{Fe}(\mathrm{III}), \mathrm{Sc}(\mathrm{III}))$. PCN-333 exhibits the largest cage $(5.5 \mathrm{~nm})$ and one of the highest void volumes $\left(3.84 \mathrm{~cm}^{3} \mathrm{~g}^{-}\right)$ among all reported MOFs. PCN-333( $\mathrm{Al}, \mathrm{Fe})$ shows high stability in aqueous solutions with $\mathrm{pH}$ values ranging from 3 to 9 , making it an extraordinary candidate for enzyme encapsulation. Three enzymes with different sizes, horseradish peroxidase (HRP), cytochrome $c$ (Cyt $c$ ) and microperoxidase-11 (MP-11), were selected. From a pure size-match point of view, HRP and Cyt $c$ will most likely undergo SEE, whereas MP-11 will go through MEE due to size mismatch. Nevertheless, all three enzymes have been successfully encapsulated into PCN-333(Al) with extremely

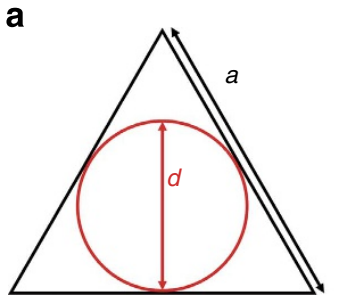

Triangle

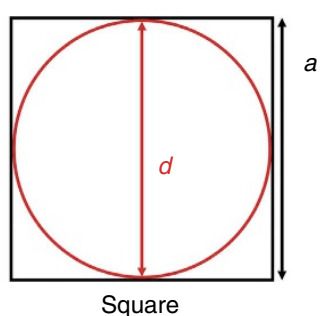

Square

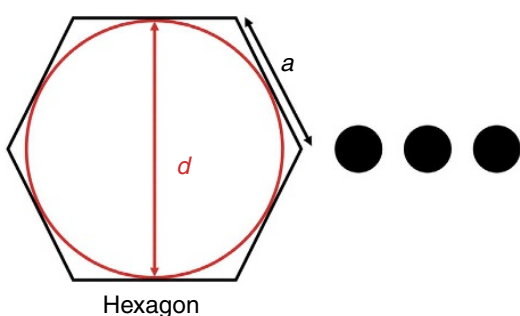

Hexagon

b
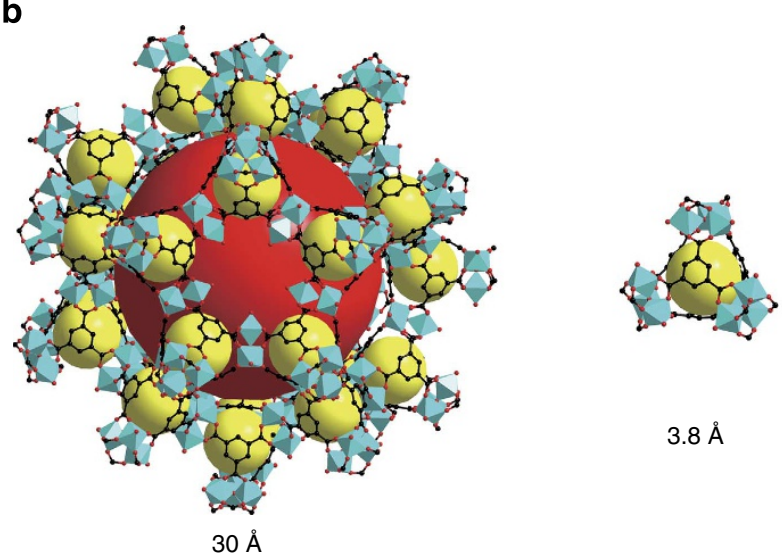<smiles>O=C1Oc2cc(C(=O)[O-])cc3c2C(O1)OC3=O</smiles>

$3.8 \AA$

$2.88 \AA$

C

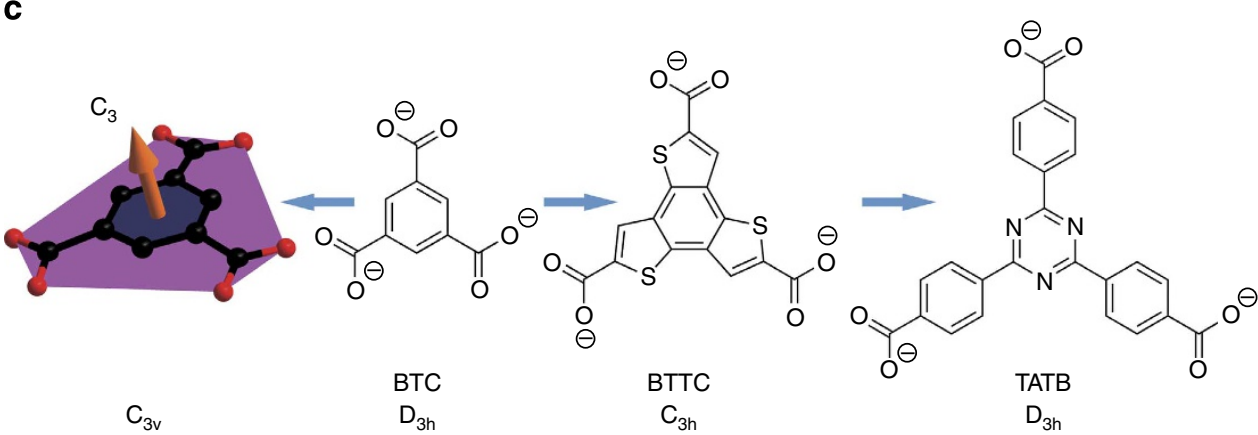

Figure 1 | Pore expansion with preserved structure (the isoreticular approach). (a) The relationship between the edge ( $a$ ) and diameter ( $d$ ) in regular polygons. (b) The largest cage, super tetrahedral unit and organic linker in MIL-100. (c) Idealized symmetries of benzenetricarboxylate (BTC) in MIL-100 and free BTC, BTTC and TATB. 
high loading amounts among all reported solid supports. These immobilized enzymes either maintain or surpass their catalytic activities over the free enzymes, and exhibit smaller $K_{\mathrm{m}}$ and better catalytic performance in organic solvents. These immobilized enzymes show almost no leaching during catalysis and recycling, and maintain high catalytic activity.

\section{Results}

Custom-designed synthesis of PCN-332 and PCN-333. The largest pore reported in MOFs was based on a $1 \mathrm{D}$ hexagonal channel structure, which requires a complicated synthetic process to make an extremely long organic linker to reach enzymatic size $^{3}$. Considering the structural feature of the $1 \mathrm{D}$ channel, it can only partially interact with immobilized enzymes even if the size is very compatible. As is in mesoporous silica, once the enzymes are incorporated, aggregation still exists. Moreover, after those channels are occupied, there is no other pathway allowing the fast diffusion of both reactants and products. In comparison, cages will act as SMTs affording strong interactions between the cages and the encapsulated enzymes. In addition, cages usually have small windows, which can efficiently encapsulate enzymes, diminishing aggregation, eliminating leaching and optimizing the catalytic environment. Unfortunately, there are very few reported MOFs with enzyme compatible cages, and even fewer can survive in aqueous media, $3,11,12$. Thus, MOFs with larger cages and better stability are highly desired for enzyme encapsulation.

The extension of organic linkers, which mostly serve as the faces or edges of pores in MOFs, is the main approach to achieve large pores in MOFs ${ }^{3,13-19}$. However, the pore shape or symmetry plays a very important role in determining the overall pore size. Approximating the cross-section of a pore (cage or channel) as a regular polygon with $n$ edges (Fig. 1a), the diameter $d$ of the inscribed circle corresponding to the pore can be expressed as the following,

$$
d=\frac{1}{\tan \left(\frac{\pi}{n}\right)} \times a
$$

where $a$ is the edge of the regular polygon.

If $a$ is considered as the edge of a pore cross-section, then $1 / \tan (\pi / n)$ would be the magnification factor, which increases with $n$ and determines how a certain change of $a$ affects the overall pore diameter $d$. Therefore, by applying the isoreticular approach through ligand extension in MOFs, the pores with larger $n$ values can be more efficiently enlarged than the pores with a smaller $n$ value.

Hence, to construct MOFs with ultra-large cages and excellent chemical stability, we applied the isoreticular approach to the chemically highly stable mesoporous MOF, MIL-100 (ref. 20). The circular cross-section of the largest cage in MIL-100 is surrounded by 12 supertetrahedra, therefore $n$ is very large $(n=12)$. In addition, MIL-100 is based on hard Lewis acidic trivalent metal species $\left(\mathrm{Al}^{3+}, \mathrm{Fe}^{3+}, \mathrm{Cr}^{3+}, \mathrm{V}^{3+}, \mathrm{Sc}^{3+}\right)$, which interact strongly with carboxylates and give rise to the high stability of the framework in aqueous environments ${ }^{21-25}$.

Benzenetricarboxylate (Fig. 1b), the organic linker in MIL-100, has idealized $D_{3 \mathrm{~h}}$ symmetry as a free anion. In the framework, the ligand symmetry is reduced to $C_{3 \mathrm{v}}$ by a bowl-shaped bending that increases the inherent energy of the ligand (the distance between the two planes is $0.288 \AA$; Fig. 1c, left). If the symmetry of the free linker were not $D_{3 \mathrm{~h}}$, to form a similar structure, the energy of the linker would have been even higher in the framework, making the overall structure extremely energetically unfavored. To guarantee the formation of isoreticular structures of MIL-100, we selected two ligands that are most stable in a trigonal planar conformation, BTTC (benzo-tris-thiophene carboxylate) with a $C_{3 \mathrm{~h}}$ (pseudo- $D_{3 \mathrm{~h}}$ in crystallography) symmetry through the fused five-member ring and TATB (4,4',4"-s-triazine-2,4,6-triyl-tribenzoate), with idealized $D_{3 \mathrm{~h}}$ symmetry arised from ligand planarity, which is enforced by conjugation (Fig. 1c).

Solvothermal reactions of $\mathrm{H}_{3}$ BTTC or $\mathrm{H}_{3}$ TATB with $\mathrm{MCl}_{3}$ $(\mathrm{M}=\mathrm{Al}, \mathrm{Fe}, \mathrm{V}, \mathrm{Sc}, \mathrm{In})$ afforded octahedral crystals of PCN332(M) $(\mathrm{M}=\mathrm{Al}, \mathrm{Fe}, \mathrm{Sc}, \mathrm{V}, \mathrm{In})$ or PCN-333(M) $(\mathrm{M}=\mathrm{Al}, \mathrm{Fe}, \mathrm{Sc})$, respectively (Fig. 2a, Supplementary Figs 1-16, Supplementary Data 1). High-resolution synchrotron powder X-ray diffraction (PXRD) collected at 17-BM, Argonne National Laboratory shows that PCN-333 is cubic with $a \approx 127 \AA$. This was further confirmed by three-dimensional rotation electron diffraction (Supplementary Methods and Supplementary Figs 17-20). The space group of $\mathrm{PCN}-333(\mathrm{Al})$ was determined to be $F d-3 m$ by combining rotation electron diffraction and high-resolution transmission electron microscopy (HRTEM; Fig. 2c, Supplementary Methods, Supplementary Figs 20,21). Structural models of PCN-333 and PCN-332 were built based on the isoreticular structure of MIL-100. The structural models of PCN$333(\mathrm{Al})$ and $\mathrm{PCN}-332(\mathrm{Fe})$ were further refined against the

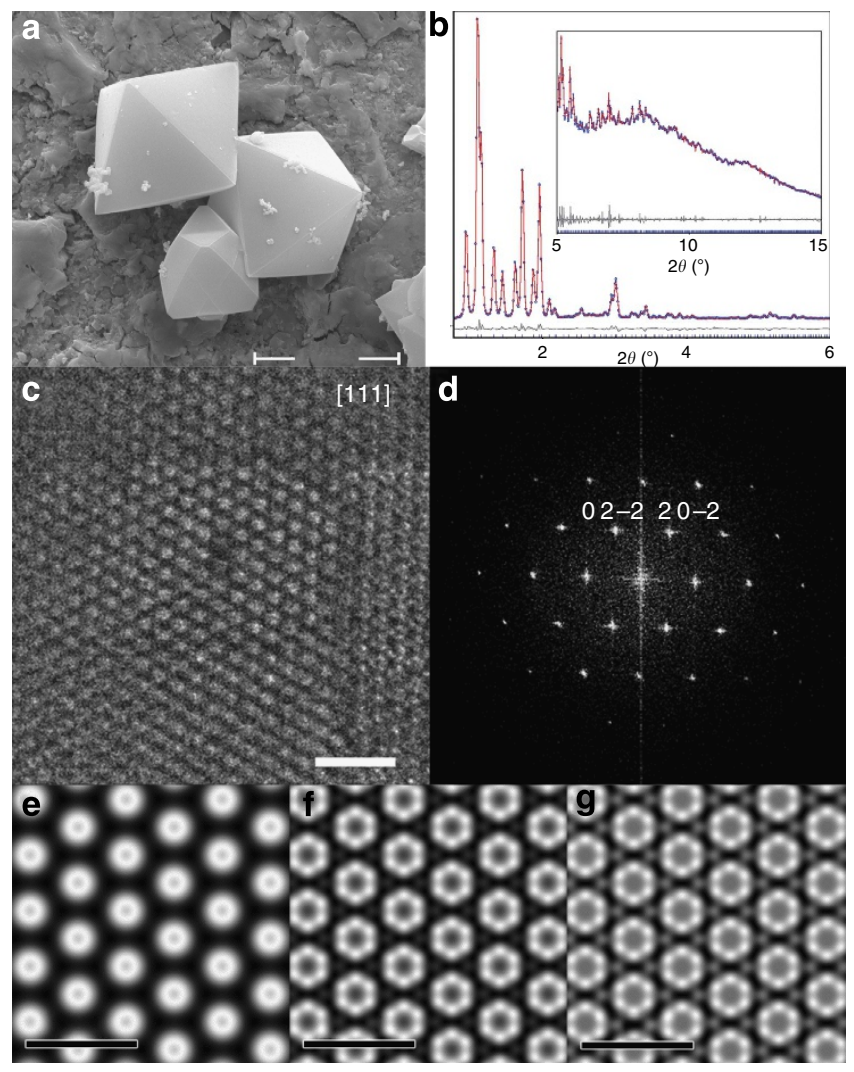

Figure 2 | Transmission- and scanning electron microscopy anlyses of PCN-333(Al). (a) SEM image of PCN-333(Al). Scale bar, $10 \mu \mathrm{m}$; (b) observed (blue), calculated (red) and difference (black) plot for the Rietveld refinement of PCN-333(Al); (c) HRTEM image of PCN-333(Al) taken along the [111] direction, showing the pore with the size of about $3.7 \mathrm{~nm}$ in projection. Scale bar, $20 \mathrm{~nm}$; (d) Fourier transform of HRTEM image in c, from which the amplitudes and phases were extracted; (e) Symmetryaveraged map after imposing the $p 6 \mathrm{~mm}$ symmetry on the amplitudes and phases; (f) Symmetry-averaged map $(p 6 \mathrm{~mm})$ calculated using the amplitudes from PXRD and phases from the HRTEM image; (g) Projected electrostatic potential map calculated using the structure factor amplitudes and the phases from the structural model. Scale bars in $\mathbf{e}-\mathbf{g}, 10 \mathrm{~nm}$. 
synchrotron PXRD data by Rietveld refinement with soft restraints for the $\mathrm{M}-\mathrm{O}$ bond distances and rigid body for the ligands (Fig. 2b and Supplementary Tables 1,2, Supplementary Fig. 5). A two-dimensional map is calculated by an inverse Fourier transformation from the amplitudes and phases of five symmetry-independent reflections with $d>14 \AA$ extracted from the HRTEM image (Fig. 2c,e), in which channels along the [111] direction with a size around $3.7 \mathrm{~nm}$ can be clearly visualized. Owing to the beam damage of the sample and the damping effect of the objective lens (contrast transfer function), amplitudes obtained from the HRTEM image are attenuated. Replacing the amplitudes from the HRTEM image by those from the PXRD pattern gives an improved two-dimensional map (Fig. 2f), which matches better the projected potential map of $\mathrm{PCN}-333(\mathrm{Al})$ calculated from the structural model (Fig. $2 \mathrm{~g}$ ). This strongly indicates that the structural model is correct.

The structure of PCN-333 is built by sharing the vertices of the supertetrahedra, which consist of $\mathrm{M}_{3}\left(\mu_{3}-\mathrm{O}\right)(\mathrm{OH})\left(\mathrm{H}_{2} \mathrm{O}\right)_{2}$ units linked along the faces by the organic linkers (Fig. 3a). Consequently, two types of mesoporous cages, which can act as SMTs for encapsulation of an enzyme, are generated. A smaller dodecahedral cage is built of 20 supertetrahedra that are connected via vertex sharing to construct a cavity with an exclusive pentagonal window of $25.9 \AA$ in diameter (Fig. 3b). A larger hexacaidecahedral (hexagonal-truncated trapezohedral) cage is surrounded by 24 supertetrahedra that form a sphere with not only the pentagonal windows, but also hexagonal windows with a diameter of $30 \AA$ (Fig. 3b). The inner diameter is $11 \AA$ for the super tetrahedral cage, $34 \AA$ for the dodecahedral cage and $55 \AA$ for the hexacaidecahedral cage (Fig. 3b). The largest cages in PCN-333 lie in a honeycomb arrangement in the [111] projection, which is consistent with the HRTEM result (Fig. 2). PCN-332 exhibits a similar structure as PCN-333, but with smaller cages due to the smaller linker (Fig. 3, Supplementary Methods).
Porosity and stability characterization. Owing to the small ligand size and strong coordination bond between the organic linker and metal struts, both the PCN-332 and the PCN-333 series can be activated directly on the removal of solvent, while many other MOFs with such high porosity must be carefully activated with supercritical $\mathrm{CO}_{2}$ (refs 10,15-18). To assess the porosity of PCN-332 and PCN-333, we performed Ar sorption at $87 \mathrm{~K}$ and $\mathrm{N}_{2}$ sorption at $77 \mathrm{~K}$ (Fig. 4a,b and Supplementary Figs 22-31 and Supplementary Methods). PCN-333(Al) shows a total Ar sorption of $2,950 \mathrm{~cm}^{3} \mathrm{~g}^{-1}$ and $\mathrm{N}_{2}$ uptake of $2,490 \mathrm{~cm}^{3} \mathrm{~g}^{-1}$. The Brunauer-Emmett-Teller surface area is $4,000 \mathrm{~m}^{2} \mathrm{~g}^{-1}$. Two steep increases at $p / p_{0}=0.3$ and 0.5 on the Ar adsorption isotherm correspond to two types of mesoporous cages in PCN-333(Al). The experimental void volume of PCN$333(\mathrm{Al})$ is $3.81 \mathrm{~cm}^{3} \mathrm{~g}^{-1}$, which is in good agreement with that calculated from the structural model $\left(3.85 \mathrm{~cm}^{3} \mathrm{~g}^{-1}\right)$. Such high porosity is comparable to those of the most porous MOFs ${ }^{13,16-}$ $19,26,27$. The porosity of PCN-333(Fe) is similar to that of PCN$333(\mathrm{Al})$, while the porosity of PCN-333(Sc) is slightly lower due to its poorer crystallinity. With an isoreticular structure but a smaller organic linker, the porosity and Brunauer-Emmett-Teller surface area of PCN-332 are significantly lower than those of PCN-333.

Despite its high porosity, PCN-333 still shows excellent stability in aqueous solutions. PXRD shows that PCN-333(Al) and PCN$333(\mathrm{Fe})$ remain intact without loss of crystallinity after immersion in pure $\mathrm{H}_{2} \mathrm{O}$ and in aqueous solutions with $\mathrm{pH}$ of 3 and 9 (Fig. 4d,f). Furthermore, the $\mathrm{N}_{2}$ sorption isotherms are almost completely maintained through those treatments (Fig. 4c,e), which further demonstrates the stability of these frameworks. The high positive charge density $(Z / r)$ on small $\mathrm{M}^{3+}\left(\mathrm{Al}^{3+}, \mathrm{Fe}^{3+}\right)$ ions causes a strong electrostatic interaction between metal nodes and carboxylate linkers, which endows the frameworks with not only excellent stability towards attack by reactive species, but also high thermal stability (Supplementary Figs 32-39).

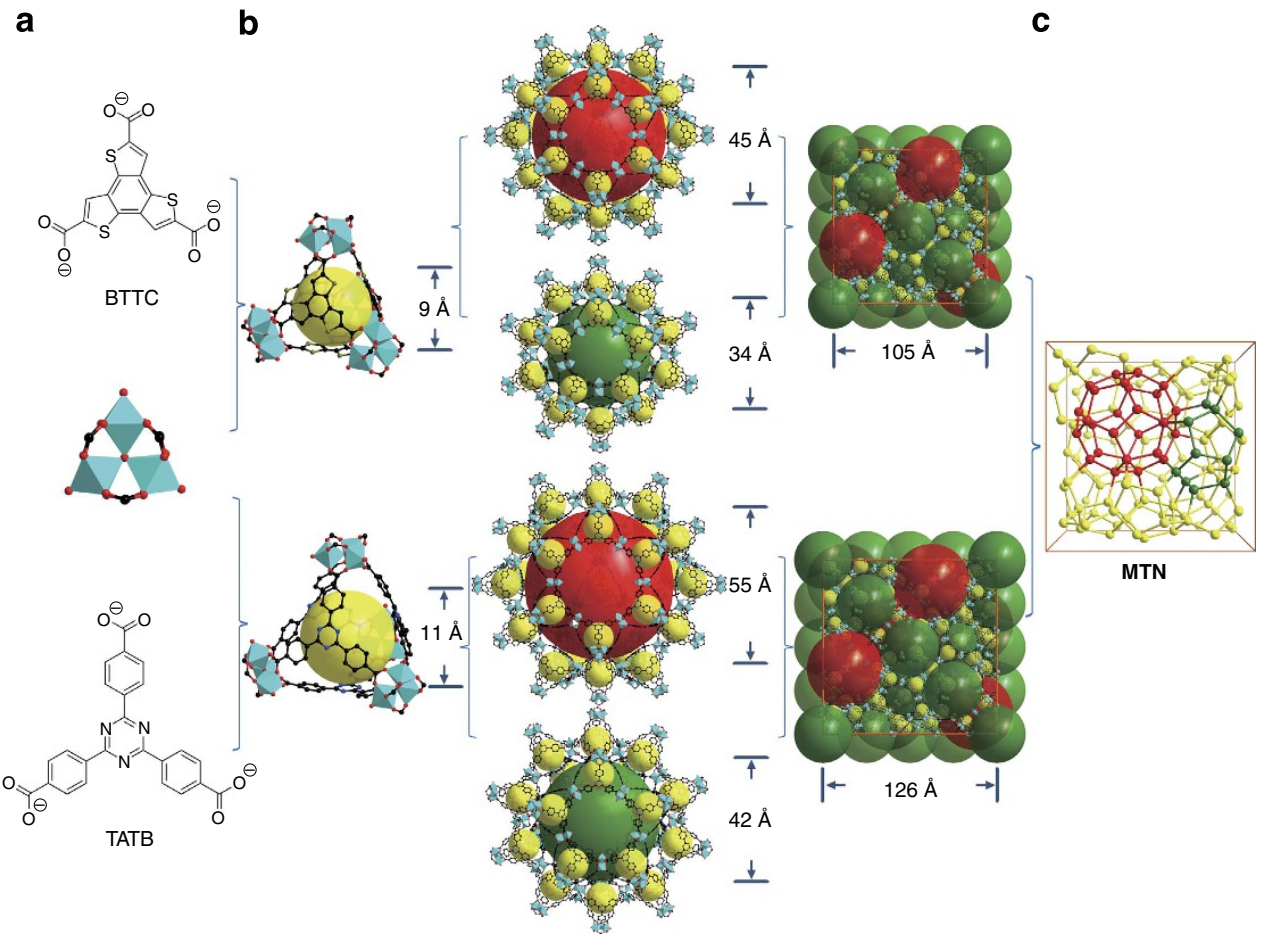

Figure 3 | Structure illustrations of PCN-332 and PCN-333. (a) Ligands used in PCN-332 and PCN-333. (b) Three different cages in PCN-332 and PCN333. (c) Simplification of PCN-332 and PCN-333 into MTN topology. 
a

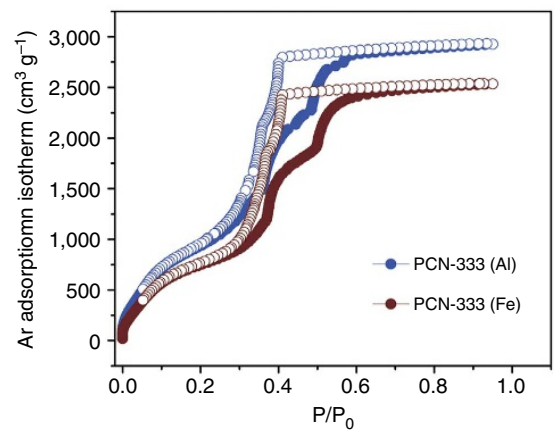

C

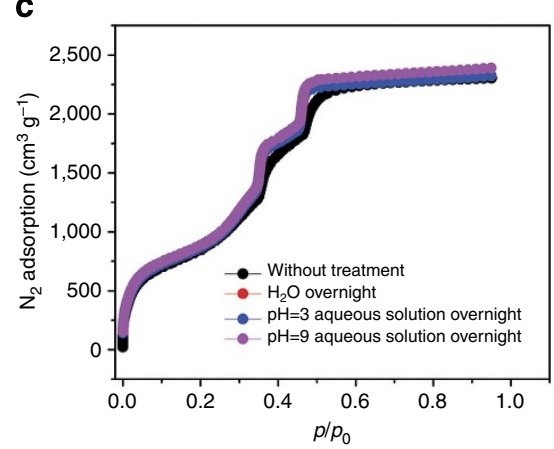

e

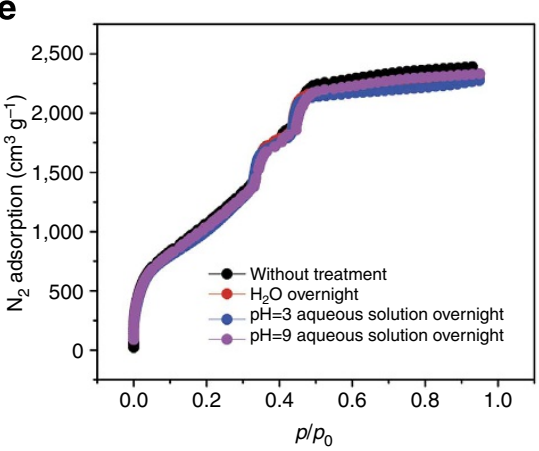

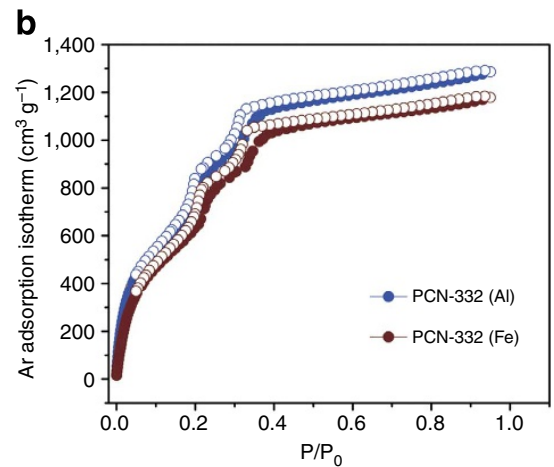

d

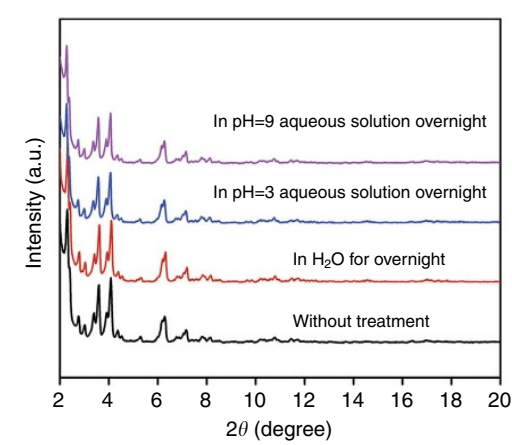

f

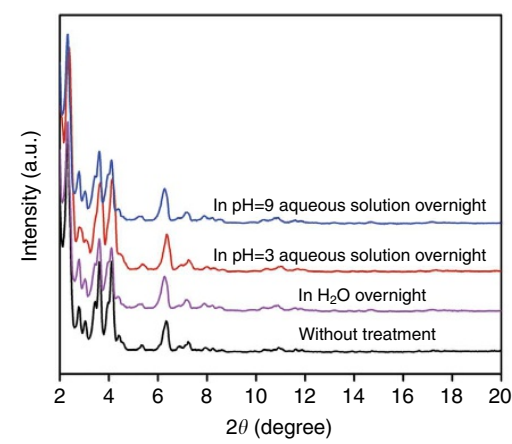

Figure 4 | Porosity and stability characterization of PCN-333 and PCN-332. (a) Ar sorption isotherms of PCN-333(Fe) and PCN-333(Al); (b) Ar sorption isotherms of PCN-332(Fe) and PCN-332(Al); (c,d) Stability tests of PCN-333(Al); (e,f) Stability tests of PCN-333(Fe).

PCN-333(Al) as support for different enzymes. PCN-333 is an extraordinary example that meets most prerequisites as a promising enzyme support: the ultra-large cavities can act as SMTs for enzyme encapsulation; the ultra-high porosity ensures high enzyme content after loading which guarantees the overall catalytic efficiency; and the SMTs can efficiently encapsulate enzymes, which can prevent both enzyme aggregation and leaching. In addition, it possesses excellent stability and is compatible with the working environment of an enzyme. A wide variety of enzymes, if smaller than the largest cage $(5.5 \mathrm{~nm})$ in $\mathrm{PCN}-333$, can in principle be immobilized ${ }^{5}$. If the enzyme is smaller than the cage, but the cage cannot accommodate two enzymes due to size limitation, a SEE can be achieved.

We have selected three commercially available enzymes that are smaller than the largest cage in PCN-333(Al)-HRP, Cyt $c$ and MP-11-for the encapsulation experiment and their catalytic performance and recyclability were also investigated.

To the best of our knowledge, all three enzymes have reached record-high loading among all enzyme immobilization cases $^{5}$. The maximum loading is $1.0 \mathrm{gg}^{-1}\left(22.7 \mu \mathrm{molg}^{-1}\right)$ for HRP, $0.95 \mathrm{gg}^{-1}\left(77.0 \mu \mathrm{molg}^{-1}\right)$ for Cyt $c$ and $0.89 \mathrm{gg}^{-1}$ $\left(478 \mu \mathrm{molg}^{-1}\right)$ for MP-11 (Fig. 5a,b and Table 1). Taking into account the size of each enzyme, HRP (size: $4.0 \times 4.4 \times 6.8 \mathrm{~nm}$ ) can only be loaded into the large cage, while Cyt $c$ (size: $2.6 \times 3.2 \times 3.3$ ) is suitable for both the large cage and the medium cage and the small cage with an inner diameter of $1.1 \mathrm{~nm}$ will be empty, allowing diffusion of solvent molecules, reactants and products. Assuming SEE, the maximum theoretical loading is $1.27 \mathrm{gg}^{-1}$ for HRP (assuming only the large cage acts as a SMT) and $1.04 \mathrm{gg}^{-1}$ for Cyt $c$ (assuming both the large and medium cages act as SMTs), which are in good agreement with the experimental maximum loadings (Supplementary Methods). For MP-11, considering its much smaller size $(1.1 \times 1.7 \times 3.3 \mathrm{~nm})$, multiple enzymes may enter each meso-cage, leading to a much higher molar concentration than the other two enzymes.

Due to the favourable interaction between the cage and the enzyme, to reach a saturated loading of each enzyme in PCN333(Al) is rapid. HRP takes about $40 \mathrm{~min}$, Cyt $c$ takes $30 \mathrm{~min}$, while MP-11 takes about $10 \mathrm{~min}$. However, for mesoporous silica, saturated enzyme loading usually takes at least several days to reach $^{28-30}$. After the loading of enzymes, the crystallinity, porosity and thermal stability of PCN-333(Al) samples were characterized, 
a

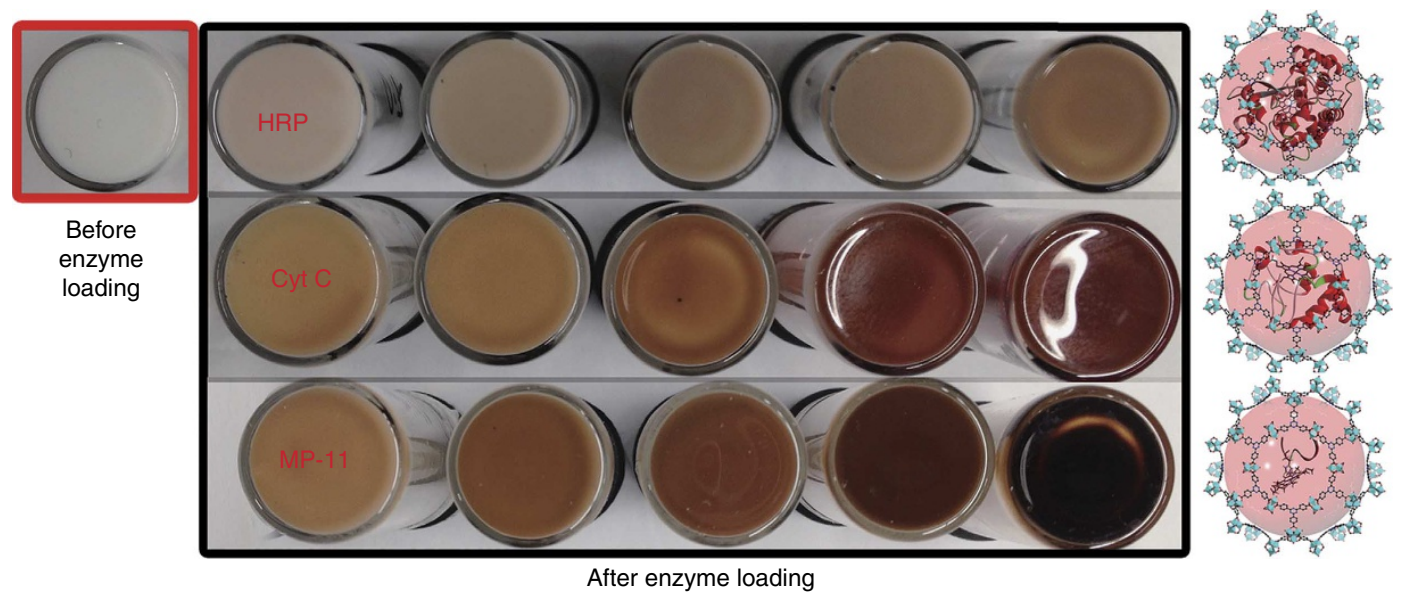

b
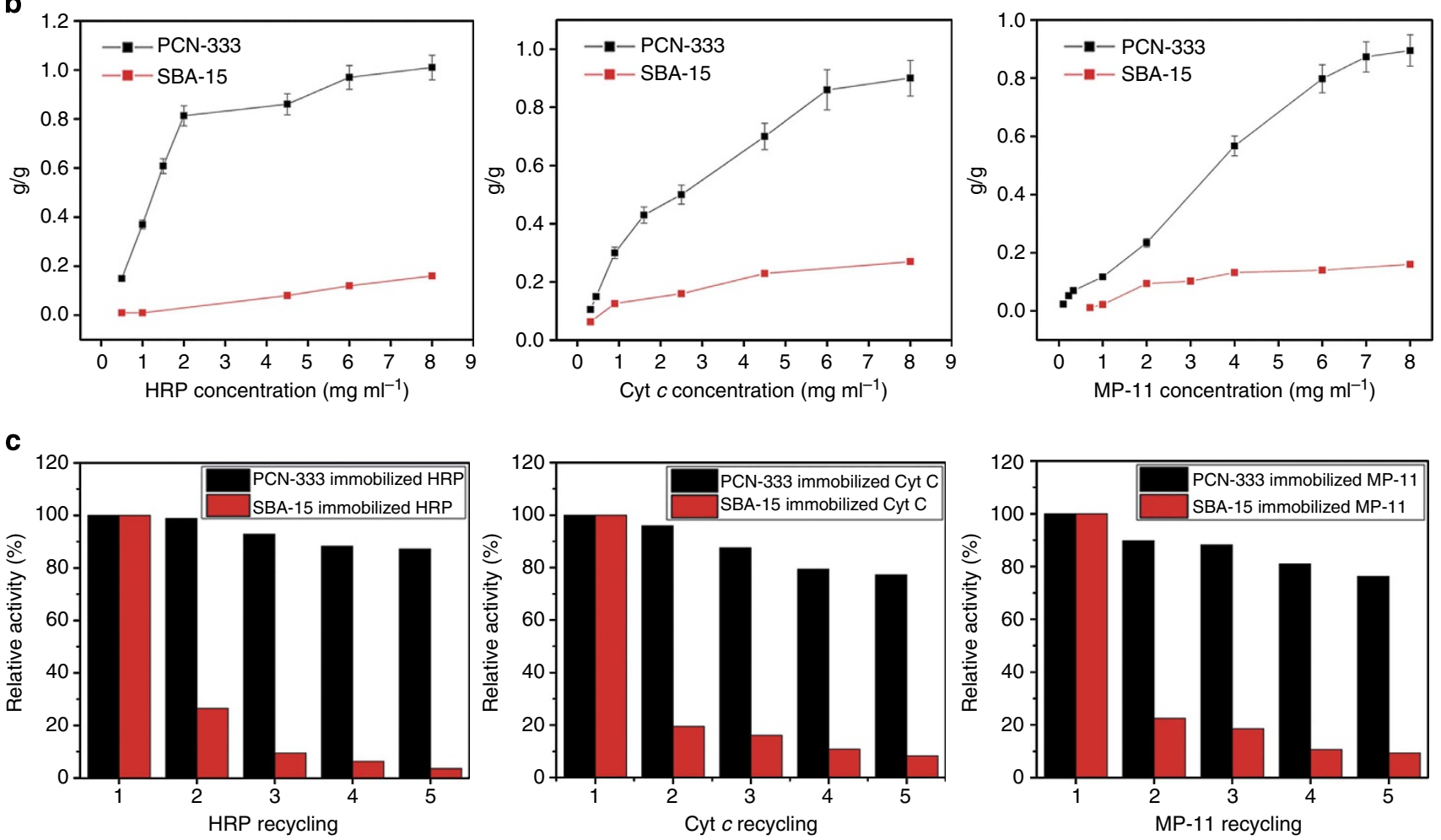

Figure 5 | Enzyme loading and catalytic recycles of different enzymes. (a) Colour variations of PCN-333(Al) when loaded with different enzymes at different concentrations. (b) Plots of the loading capacities of different enzymes in PCN-333(AI). The standard deviation (error bars) was obtained from three independent replicate experiment. (c) Catalytic activity of immobilized enzymes in each recycle test.

showing well-maintained framework (Supplementary Figs 40-54). Most importantly, the transmission electron microscopy-energydispersive X-ray spectroscopy elemental mapping of HRP@PCN333(Al) sample indicates very well distributed HRP inside of the framework (Supplementary Fig. 67).

Enzyme leaching and loss of reactivity on reuse are the major problems for all other enzyme supports ${ }^{5}$. After the immobilization of the three enzymes in PCN-333(Al), their catalytic activity was then evaluated with corresponding reactions (Supplementary Figs 55-74).

\section{Discussion}

Oxidation of o-Phenylenediamine catalysed by HRP and oxidation of 2-2'-azino-bis(3-ethylbenzthiazoline-6-sulfonic acid) catalysed by Cyt $c$ and MP-11 were performed, respectively, in acid-sodium citrate buffer with $\mathrm{pH}=6$ at room temperature. Compared with the free enzymes, enzymes immobilized in $\mathrm{PCN}-333(\mathrm{Al})$ show relatively smaller $k_{\text {cat }}$ values, which may be caused by diffusion control. However, all three enzymes exhibit smaller $K_{\mathrm{m}}$ values, resulting in comparable $k_{\text {cat }} / K_{\mathrm{m}}$ values to the free enzymes (Table 2). The low $K_{\mathrm{m}}$ means that the enzyme requires a lower substrate concentration to achieve $V_{\max }$ and usually suggests that the enzyme may have a higher affinity with the substrate.

The free enzyme reacts more quickly than the encapsulated enzymes, though it requires significantly more concentration of substrate to reach its peak rate, under carefully controlled conditions designed to prevent it from being aggregated or denatured. However, in practical application, the reaction usually needs to be conducted in different media other than the buffer 


\section{Table 1 | Comparison of different enzyme loadings in PCN-333(Al) and SBA-15.}

\begin{tabular}{|c|c|c|c|}
\hline Enzyme & $\begin{array}{l}\text { Max-loading in } \\
\text { PCN-333(Al) } \\
\left(\mathrm{gg}^{-1}\right)\end{array}$ & $\begin{array}{l}\text { Max-loading in } \\
\text { PCN-333(Al) } \\
\left(\mu \mathrm{molg}^{-1}\right)\end{array}$ & $\begin{array}{c}\text { Max-loading in } \\
\text { SBA-15 } \\
\left(\mu \mathrm{mol} \mathrm{g}^{-1}\right)\end{array}$ \\
\hline HRP & 1.0 & 22.7 & 4.2 \\
\hline Cyt c & 0.95 & 77.0 & 45 \\
\hline MP-11 & 0.89 & 478 & 20 \\
\hline
\end{tabular}

Cyt c, cytochrome c; HRP, horseradish peroxidase; MP-11, microperoxidase-11.

\begin{tabular}{|c|c|c|c|}
\hline Enzymes & $K_{m}(m M)$ & $k_{\text {cat }}\left(\min ^{-1}\right)$ & $\begin{array}{c}k_{\text {cat }} / K_{m} \\
\left(\mathrm{~min}^{-1} \mathbf{m M}^{-1}\right)\end{array}$ \\
\hline \multicolumn{4}{|l|}{$H R P$} \\
\hline PCN-333 immobilized & 0.84 & $1.54 \times 10^{4}$ & $1.833 \times 10^{4}$ \\
\hline Free & 1.8 & $4.52 \times 10^{4}$ & $2.51 \times 10^{4}$ \\
\hline \multicolumn{4}{|l|}{ Cyt c } \\
\hline PCN-333 immobilized & 14.9 & 32.2 & 2.16 \\
\hline Free & 48.3 & 65.8 & 1.36 \\
\hline \multicolumn{4}{|l|}{ MP-11 } \\
\hline PCN-333 immobilized & 2.15 & 27.9 & 12.98 \\
\hline Free & 3.28 & 80.2 & 24.45 \\
\hline
\end{tabular}

Cyt c, cytochrome c; HRP, horseradish peroxidase; MP-11, microperoxidase-11.

solution. Therefore, we measured the specific activity to investigate the catalytic activity of SEE and MEE in different media. Cyt $c$, which is of a suitable size to be incorporated into the two kinds of mesocages in a 1:1 ratio, has most likely undergone SEE, and shows 14 times higher specific activity after immobilization in PCN-333(Al) than the free enzyme in water. To provide a more comprehensive comparison about the immobilized and free Cyt $c$, the kinetic parameters were investigated in this condition and the results indicate the immobilized Cyt $c$ has a smaller $K_{\mathrm{m}}$, and a higher $K_{\text {cat }}$ and $K_{\text {cat }} / K_{\mathrm{m}}$ in water media (Table 3 ). Considering that Cyt $c$ suffers severe self-aggregation or denaturation towards temperature or $\mathrm{pH}$ variation ${ }^{31}$, such significant catalytic activity enhancement is evidence of SEE in PCN-333(Al). Immobilized HRP, which can also realize SEE, shows slightly higher specific activity (Supplementary Fig. 68) in water. In comparison, MP-11, the size of which is much smaller than the mesocages, suitable for MEE, shows much decreased activity after immobilization (Supplementary Fig. 68). Moreover, in several different organic solvent mixtures, the immobilized HRP performs better than the free HRP (Supplementary Fig. 71). These results show the effectiveness of SEE under harsh reaction conditions.

After the completion of each catalytic reaction, the immobilized enzymes were then recycled by a simple centrifuge. The catalytic reactivity remains almost unchanged for each PCN333(Al) encapsulated enzyme after several cycles, while very significant decrease of the activity is observed for the same enzymes immobilized in SBA-15, a well-known type of mesoporous silica (Fig. 5c). The main reason for the excellent recyclability of PCN-333(Al) immobilized enzymes is the SEE or MEE of these enzymes: there is almost no leaching from these encapsulated enzymes during many cycles while high apparent
Table 3 | Comparison of kinetic parameters for free and immobilized Cyt $c$ in aqueous media.

\begin{tabular}{lccc} 
& $\boldsymbol{K}_{\mathbf{m}} \mathbf{( m M )}$ & $\boldsymbol{k}_{\text {cat }}\left(\mathbf{m i n}^{-\mathbf{1})}\right.$ & $\begin{array}{c}\boldsymbol{k}_{\mathbf{c a t}} / \boldsymbol{K}_{\mathbf{m}} \\
\left(\mathbf{m i n}^{-\mathbf{1}} \mathbf{m M} \mathbf{- 1}\right)\end{array}$ \\
\hline PCN-333-immobilized Cyt C & 6.8 & 197 & 28.97 \\
Free Cyt c & 109.4 & 109 & 1.00 \\
\hline Cyt c, cytochrome c. & & & \\
\hline
\end{tabular}

leaching is observed in SBA-15. Leaching rate is usually thermodynamically related to the interaction strength between the enzyme and the support ${ }^{5}$. Physisorption is usually much weaker than covalent immobilization. However, when there are many interaction sites such as in the case of encapsulation, the overall interaction strength can be stronger than a single covalent bond. Each large cage in PCN-333 is surrounded by many supertetrahedra containing not only highly concentrated metal sites with polarized coordinating water molecules, but also conjugated organic linkers, which generate an amphiphilic inner sphere inside the cage. The cage can therefore act as a SMT, providing a strong trapping interaction to both the hydrophilic and hydrophobic parts of the enzyme, which explains the fast enzyme uptake and the negligible enzyme leaching after encapsulation. The strong interaction between the enzyme and the cages in PCN-333 indicates a large equilibrium constant if the loading process is considered as a reaction. The loading process is less reversible, which leads to fast completion of the forward reaction (enzyme loading). In addition, the small window on each large cage can dynamically reduce the enzyme leaching. In comparison, SBA-15, which has 1D channels with fully hydrophilic surfaces, can provide only limited interaction to immobilized enzymes and lead to very significant enzyme leaching during catalysis and recycling processes.

After the encapsulation of enzymes in PCN-333(Al), enzymatic catalysis in organic solvents, an important field in the biotechnological applications of proteins ${ }^{32}$, can be conducted more easily by overcoming the solubility issues for both enzymes in organic media and substrates in aqueous media. The encapsulated HRP shows higher catalytic activity in tetrahydrofuran-water media than it does in pure water, and much higher activity than free HRP under the same condition (Supplementary Methods).

In summary, by using an exceptionally water-stable MOF containing mesocages that can act as single-molecule traps for the encapsulation of a single-enzyme molecule in one cage, essentially eliminating enzyme aggregation and leaching, immobilized enzymes with recyclability and high catalytic efficiency in harsh condition have been discovered. The single-enzyme encapsulation concept will revolutionize the application of enzymes in industry, enabling the application of enzymes in fundamental processes such as nitrogen fixation, hydrogen production and the conversion of natural gas into liquid fuels of high energy density with unprecedented efficiency.

\section{Methods}

Synthesis of PCN-332(Al). $\mathrm{H}_{3} \mathrm{BTTC}(50 \mathrm{mg})$ and $\mathrm{AlCl}_{3} 6 \mathrm{H}_{2} \mathrm{O}(200 \mathrm{mg})$ were dissolved in $10 \mathrm{ml}$ diethylformamide (DEF) or dimethylformamide (DMF), then $1.5 \mathrm{ml}$ trifluoroacetic acid was added. The mixture was heated up at $150{ }^{\circ} \mathrm{C}$ in an oven for $12 \mathrm{~h}$ until white precipitate formed. The white precipitate was centrifuged and washed with fresh DMF for several times. Yield (based on ligand): $\sim 70 \%$.

Synthesis of PCN-332(Fe). $\mathrm{H}_{3}$ BTTC (50 mg) and anhydrous $\mathrm{FeCl}_{3}(60 \mathrm{mg}$ ) were dissolved in $10 \mathrm{ml}$ DEF or DMF, then $1.2 \mathrm{ml}$ trifluoroacetic acid was added. The mixture was heated up at $150^{\circ} \mathrm{C}$ in an oven for $12 \mathrm{~h}$ until brown precipitate formed. The brown precipitate was centrifuged and washed with fresh DMF for several times. Yield (based on ligand): $\sim 85 \%$. 
Synthesis of PCN-332(V). $\mathrm{H}_{3}$ BTTC $(50 \mathrm{mg})$ and anhydrous $\mathrm{VCl}_{3}(60 \mathrm{mg})$ were dissolved in $10 \mathrm{ml} \mathrm{DEF}$ or DMF. The mixture was heated up at $150^{\circ} \mathrm{C}$ in an oven for $4 \mathrm{~h}$ until green precipitate formed. The green precipitate was centrifuged and washed with fresh DMF for several times. Yield (based on ligand): $\sim 75 \%$.

Synthesis of PCN-332(Sc). $\mathrm{H}_{3} \mathrm{BTTC}(50 \mathrm{mg})$ and $\mathrm{ScCl}_{3} 6 \mathrm{H}_{2} \mathrm{O}(200 \mathrm{mg})$ were dissolved in $10 \mathrm{ml}$ DEF or DMF. The mixture was heated up at $150^{\circ} \mathrm{C}$ in an oven for $4 \mathrm{~h}$ until white precipitate formed. The white precipitate was centrifuged and washed with fresh DMF for several times. Yield (based on ligand): $\sim 80 \%$.

Synthesis of PCN-332(In). $\mathrm{H}_{3} \mathrm{BTTC}(10 \mathrm{mg})$ and $\mathrm{In}\left(\mathrm{NO}_{3}\right)_{3} \mathrm{xH}_{2} \mathrm{O}(20 \mathrm{mg})$ were dissolved in $2 \mathrm{ml} \mathrm{DMF}$, then $0.2 \mathrm{ml} \mathrm{HNO}_{3}(3 \mathrm{M}$ in DMF) was added. The mixture was heated up at $150^{\circ} \mathrm{C}$ in an oven for $12 \mathrm{~h}$. The white precipitate was centrifuged and washed with fresh DMF for several times. Yield (based on ligand): $\sim 85 \%$.

Synthesis of PCN-333(Al). $\mathrm{H}_{3} \mathrm{TATB}(50 \mathrm{mg})$ and $\mathrm{AlCl}_{3} 6 \mathrm{H}_{2} \mathrm{O}(200 \mathrm{mg})$ were dissolved in $10 \mathrm{ml}$ DEF or DMF, then $1.0 \mathrm{ml}$ trifluoroacetic acid was added. The mixture was heated up at $135^{\circ} \mathrm{C}$ in an oven for 2 days until white precipitate formed. The white precipitate was centrifuged and washed with fresh DMF for several times. Yield (based on ligand): $80 \%$.

Synthesis of PCN-333(Fe). $\mathrm{H}_{3}$ TATB $(50 \mathrm{mg})$ and anhydrous $\mathrm{FeCl}_{3}(60 \mathrm{mg})$ were dissolved in $10 \mathrm{ml}$ DEF or DMF, then $0.5 \mathrm{ml}$ trifluoroacetic acid was added. The mixture was heated up at $150^{\circ} \mathrm{C}$ in an oven for $12 \mathrm{~h}$ until brown precipitate formed. The brown precipitate was centrifuged and washed with fresh DMF for several times. Yield (based on ligand): $85 \%$.

Synthesis of PCN-333(Sc). $\mathrm{H}_{3}$ TATB $(50 \mathrm{mg})$ and $\mathrm{ScCl}_{3} 6 \mathrm{H}_{2} \mathrm{O}(200 \mathrm{mg})$ were dissolved in $10 \mathrm{ml}$ DEF or DMF. The mixture was heated up at $150^{\circ} \mathrm{C}$ in an oven for $4 \mathrm{~h}$ until white precipitate formed. The white precipitate was centrifuged and washed with fresh DMF for several times. Yield (based on ligand): $\sim 85 \%$.

\section{References}

1. Zhou, H.-C., Yaghi, O. M. \& Long, J. R. Introduction to metal-organic frameworks. Chem. Rev. 112, 673-674 (2012).

2. Férey, G. et al. A chromium terephthalate-based solid with unusually large pore volumes and surface area. Science 309, 2040-2042 (2005).

3. Deng, H. et al. Large-pore apertures in a series of metal-organic frameworks. Science 336, 1018-1023 (2012).

4. Lykourinou, V. et al. Immobilization of MP-11 into a mesoporous metalorganic framework, MP-11@mesoMOF: a new platform for enzymatic catalysis. J. Am. Chem. Soc. 133, 10382-10385 (2011).

5. Hudson, S., Cooney, J. \& Magner, E. Proteins in mesoporous silicates. Angew. Chem. Int. Ed. 47, 8582-8594 (2008).

6. Klibanov, A. M. Immobilized enzymes and cells as practical catalysts. Science 219, 722-727 (1983).

7. Filice, M., Guisan, J. M., Terreni, M. \& Palomo, J. M. Regioselective monodeprotection of peracetylated carbohydrates. Nat. Protoc. 7, 1783-1796 (2012).

8. Datta, S. et al. Enzyme immobilization: an overview on techniques and support materials. Biotech. 3, 1-9 (2013).

9. Engstrom, K. et al. Co-immobilization of an enzyme and a metal into the compartments of mesoporous silica for cooperative tandem catalysis: an artificial metalloenzyme. Angew. Chem. Int. Ed. 52, 14006-14010 (2013).

10. Li, J.-R. et al. Porous materials with pre-designed single-molecule traps for $\mathrm{CO}_{2}$ selective adsorption. Nat. Commun. 4, 1538 (2013).

11. Liu, W.-L. et al. Fast multipoint immobilized MOF bioreactor. Chem. Eur. J 20, 8923-8928 (2014)

12. Liu, W.-L. et al. Novel trypsin-FITC@MOF bioreactor efficiently catalyzes protein digestion. J. Mater. Chem. B 1, 928-932 (2013).

13. Furukawa, H. et al. Ultrahigh porosity in metal-organic frameworks. Science 329, 424-428 (2010).

14. Farha, O. K. et al. Metal-organic framework materials with ultrahigh surface areas: Is the sky the limit? J. Am. Chem. Soc. 134, 15016-15021 (2012).

15. Yuan, D. et al. An isoreticular series of metal-organic frameworks with dendritic hexacarboxylate ligands and exceptionally high gas-uptake capacity. Angew. Chem. Int. Ed. 49, 5357-5361 (2010).

16. Koh, K., Wong-Foy, A. G. \& Matzger, A. J. A crystalline mesoporous coordination copolymer with high microporosity. Angew. Chem. Int. Ed. 47, 677-680 (2008)

17. Farha, O. K. et al. De novo synthesis of a metal-organic framework material featuring ultrahigh surface area and gas storage capacities. Nat. Chem. 2 , 944-948 (2010).

18. Chae, H. K. et al. A route to high surface area, porosity and inclusion of large molecules in crystals. Nature 427, 523-527 (2004).

19. Furukawa, H., Cordova, K. E., O'Keeffe, M. \& Yaghi, O. M. The chemistry and applications of metal-organic frameworks. Science 341, 1230-1235 (2013).
20. Férey, G. et al. A hybrid solid with giant pores prepared by a combination of targeted chemistry, simulation, and powder diffraction. Angew. Chem. Int. Ed. 43, 6296-6301 (2004).

21. Bloch, E. D. et al. Metal insertion in a microporous metal - organic framework lined with 2,2'-bipyridine. J. Am. Chem. Soc. 132, 14382-14384 (2010).

22. Millange, F. et al. Synthesis, structure determination and properties of MIL53as and MIL-53ht: the first $\mathrm{Cr}^{\mathrm{III}}$ hybrid inorganic-organic microporous solids: $\mathrm{Cr}^{\mathrm{III}}(\mathrm{OH}) \cdot\left\{\mathrm{O}_{2} \mathrm{C}-\mathrm{C}_{6} \mathrm{H}_{4}-\mathrm{CO}_{2}\right\} \cdot\left\{\mathrm{HO}_{2} \mathrm{C}-\mathrm{C}_{6} \mathrm{H}_{4}-\mathrm{CO}_{2} \mathrm{H}\right\}_{\mathrm{x}}$. Chem. Commun. 822-823 (2002).

23. Reinsch, $\mathrm{H}$. et al. A new Al-MOF based on a unique column-shaped inorganic building unit exhibiting strongly hydrophilic sorption behaviour. Chem. Commun. 48, 9486-9488 (2012).

24. Yang, S. et al. Selectivity and direct visualization of carbon dioxide and sulfur dioxide in a decorated porous host. Nat. Chem. 4, 887-894 (2012).

25. Serre, C. et al. Role of solvent-host interactions that lead to very large swelling of hybrid frameworks. Science 315, 1828-1831 (2007).

26. An, J. et al. Metal-adeninate vertices for the construction of an exceptionally porous metal-organic framework. Nat. Commun. 3, 604 (2012).

27. Li, T. et al. Stepwise ligand exchange for the preparation of a family of mesoporous MOFs. J. Am. Chem. Soc. 135, 11688-11691 (2013).

28. Qiao, S. Z. et al. Synthesis and bio-adsorptive properties of large-pore periodic mesoporous organosilica rods. Chem. Mater. 17, 6172-6176 (2005).

29. Deere, J., Magner, E., Wall, J. G. \& Hodnett, B. K. Mechanistic and structural features of protein adsorption onto mesoporous silicates. J. Phys. Chem. B 106, 7340-7347 (2002).

30. Takahashi, H. et al. Catalytic activity in organic solvents and stability of immobilized enzymes depend on the pore size and surface characteristics of mesoporous silica. Chem. Mater. 12, 3301-3305 (2000).

31. Vazquez-Duhalt, R. Cytochrome c as a biocatalyst. J. Mol. Catal. B: Enzym. 7, 241-249 (1999)

32. Vazquez-Duhalt, R., Semple, K. M., Westlake, D. W. \& Fedorak, P. M. Effect of water-miscible organic solvents on the catalytic activity of cytochrome c. Enzyme Microb. Technol. 15, 936-943 (1993).

\section{Acknowledgements}

This work was supported as a part of the Methane Opportunities for Vehicular Energy (MOVE) Program under the Award Number DE-AR0000249, as part of the Center for Clean-Energy-Related Gas Separation, an Energy Frontier Research Center funded by the U. S. Department of Energy (DOE), Office of Science, Office of Basic Energy Sciences and as part of N000141310753 supported by the Office of Naval Research. Use of the Advanced Photon Source, an Office of Science User Facility operated for the U. S. Department of Energy (DOE) Office of Science by Argonne National Laboratory, was supported by the U. S. DOE under Contract No. DE-AC02-06CH11357. The structure characterization by PXRD and TEM was supported by the Swedish Research Council (VR), the Swedish Governmental Agency for Innovation Systems (VINNOVA) and the Knut \& Alice Wallenberg Foundation through a grant for purchasing the TEM and the project grant 3DEM-NATUR. We also thank Zhenggang Xu and Dr Qian Peng from the Supercomputing Facility at Texas A\&M University and Laboratory for Molecular Simulation for offering access to computing time and valuable discussions; Dr Hung-Jue Sue and Dr Peng Liu from Mechanical Engineering at Texas A\&M University for Thermogravimetric analysis; Dr Hansoo Kim in the Microscopy and Imaging Center at Texas A\&M University for the TEM-DEX elemental mapping; Lizzie West for organizing other manuscript related works.

\section{Author contributions}

D.F., T.-F.L and H.-C.Z. planned and executed the synthesis and characterization. J.S., W.W. and X.Z. performed the structure characterization. M.B., Z.W., Y.-P.C., X.W., K.W., X.L., Z.-Y.G., J.P. and D.Y. participated in the preparation of the manuscript.

\section{Additional information}

Accession codes: The X-ray crystallographic coordinates for structures reported in this Article have been deposited at the Cambridge Crystallographic Data Centre (CCDC), under deposition number 999752 (PCN-332(Fe)) and 997911 (PCN-333(Al)). These data can be obtained free of charge from The Cambridge Crystallographic Data Centre via www.ccdc.cam.ac.uk/data_request/cif.

Supplementary Information accompanies this paper at http://www.nature.com/ naturecommunications

Competing financial interests: The authors declare no competing financial interests.

Reprints and permission information is available online at http://npg.nature.com/ reprintsandpermissions/

How to cite this article: Feng, D. et al. Stable metal-organic frameworks containing single-molecule traps for enzyme encapsulation. Nat. Commun. 6:5979 doi: $10.1038 /$ ncomms6979 (2015). 